\title{
Evaluation of negative-pressure wound therapy for patients with diabetic foot ulcers: systematic review and meta-analysis
}

This article was published in the following Dove Press journal:

Therapeutics and Clinical Risk Management

18 April 2017

Number of times this article has been viewed

\author{
Si Liu' \\ Chao-zhu $\mathrm{He}^{\prime}$ \\ Yan-ting Cai' \\ Qiu-ping Xing' \\ Ying-zhen Guo' \\ Zhi-long Chen' \\ Ji-liang Su' \\ Li-ping Yang ${ }^{2}$
}

'School of Nursing, Nanchang University, ${ }^{2}$ Department of

Endocrinology, The Second Affiliated

Hospital of Nanchang University,

Nanchang, Jiangxi, China
Correspondence: Chao-zhu He School of Nursing, Nan Chang University, Ba yi Road, Nanchang, Jiangxi, 330006, China

Tel +86 79l 86362043

Email1028970010@qq.com
Objectives: The aim of this study was to perform an updated systematic review and meta-analysis to assess the clinical efficacy, safety, and cost-effectiveness of negative-pressure wound therapy (NPWT) in the treatment of diabetic foot ulcers (DFUs).

Methods: We searched the Cochrane Library, MEDLINE, EMBASE, Ovid, and Chinese Biological Medicine databases up to June 30, 2016. We also manually searched the articles from reference lists of the retrieved articles, which used the NPWT system in studies of vacuumassisted closure therapy. Studies were identified and selected, and two independent reviewers extracted data from the studies.

Results: A total of eleven randomized controlled trials, which included a total of 1,044 patients, were selected from 691 identified studies. Compared with standard dressing changes, NPWT had a higher rate of complete healing of ulcers (relative risk, 1.48; 95\% confidence interval $[\mathrm{CI}]: 1.24-1.76 ; P<0.001$ ), shorter healing time (mean difference, $-8.07 ; 95 \%$ CI: $-13.70--2.45 ; P=0.005$ ), greater reduction in ulcer area (mean difference, $12.18 ; 95 \% \mathrm{CI}$ : 8.50-15.86; $P<0.00001$ ), greater reduction in ulcer depth (mean difference, $40.82 ; 95 \% \mathrm{CI}$ : 35.97-45.67; $P<0.00001$ ), fewer amputations (relative risk, $0.31 ; 95 \%$ CI: $0.15-0.62 ; P=0.001$ ), and no effect on the incidence of treatment-related adverse effects (relative risk, $1.12 ; 95 \% \mathrm{CI}$ : $0.66-1.89 ; P=0.68)$. Meanwhile, many analyses showed that the NPWT was more cost-effective than standard dressing changes.

Conclusion: These results indicate that NPWT is efficacious, safe, and cost-effective in treating DFUs.

Keywords: diabetic foot ulcers, negative-pressure wound therapy, complete wound closure, amputation, meta-analysis, cost-effectiveness

\section{Introduction}

Diabetes mellitus (DM) is a syndrome characterized by hyperglycemia that results from absolute or relative impairment in insulin secretion and/or insulin action. ${ }^{1}$ With the development of people's living standards and lifestyle changes, the incidence of diabetes has been rising. An estimated 382 million people had DM in 2013; this number will increase to 592 million by $2035 .{ }^{2}$ Hazards of DM usually present as complications; diabetic foot ulcers (DFUs) are considered one of the most common and devastating chronic complications of diabetes because they contribute to high morbidity, high hospitalization rates, and high mortality, all of which seriously threaten the quality of life of DM patients. The expected lifetime risk of a DM patient developing a foot ulcer is $12 \%-25 \%,{ }^{3}$ with a $50 \%-70 \%$ recurrence rate over the ensuing 5 years. As a consequence of DFUs, a lower limb is lost every 30 seconds somewhere in the 
world, and the probability of losing the other leg is 50\% after 3 years. DFUs contribute to $85 \%$ of non-traumatic, lowerextremity amputations and lead to a 13\%-17\% mortality rate in patients with DM. ${ }^{4,5}$ In comparison to non-DFU patients, DFU patients have more days of hospitalization and more days requiring home health care, emergency department visits, and outpatient/physician office visits. ${ }^{6}$ Meanwhile, the cost of treating DFUs for complete healing and trans-tibial amputation ranges from US\$3,959 to US\$188,645 in the US. ${ }^{7}$ These numbers indicate that DFUs also impose a substantial burden on public and private payers.

The standard of care for DFUs involves debridement, local wound care, infection control, and off-loading of pressure. Various treatments advocated in recent years include advanced wound dressings, growth factors, hyperbaric oxygen therapy, cultured skin substitutes, and other wound therapies. Negative-pressure wound therapy (NPWT) is a newer, noninvasive adjunctive therapy system. A vacuum-assisted closure (VAC) device to control sub-atmospheric pressure helps promote wound healing by removing fluid from open wounds, preparing the wound bed for closure, reducing edema, and promoting formation and perfusion of granulation tissue. ${ }^{8}$ Some clinical evidence has suggested that NPWT is an effective and safe method for promoting diabetic foot wounds' healing, 9,10 but some serious complications related to NPWT have been reported in recent years. ${ }^{11}$ It is also worth noting that NPWT appears to be more expensive than conventional methods in the treatment of DFUs. Some of the previous literature focused on one or a few of the several factors of NWPT for DFUs such as evaluating efficacy, safety, and cost-effectiveness, but almost never evaluating all of them at the same time.

The aim of this study was to perform an updated systematic review and meta-analysis to assess the clinical efficacy, safety, and cost-effectiveness of NPWT in the treatment of DFUs, and to strengthen the evidence to support recommendations regarding the use of NPWT in DFU patients.

\section{Methods}

We conducted a systematic review, using the Preferred Reporting Items for Systematic Reviews and Meta-Analyses (PRISMA) checklist.

\section{Search strategy}

We searched the Cochrane Library, MEDLINE, EMBASE, Ovid, and Chinese Biological Medicine databases (up to June 30, 2016) to identify relevant reports of randomized controlled trials (RCTs) and manually searched articles from reference lists of retrieved articles to assemble a comprehensive collection of RCTs about NPWT in the treatment of DFUs.
The search terms used were "diabetic foot", "diabetic feet", "foot ulcer, diabetic", "foot, diabetic", "feet, diabetic", "negative pressure wound therapy", "negative-pressure wound therapies", "vacuum assisted closure", "vacuum-assisted closure", "topical negative pressure therapy", "negative pressure dressings", "VAC", and "NPWT" (Supplementary material).

\section{Selection criteria}

Inclusion criteria were as follows: 1) RCTs comparing NPWT (VAC) with standard dressing changes in diabetic patients; 2) diabetic patients with chronic foot ulcers and surgical foot wounds; 3) English and Chinese publication languages only; 4) diabetic patients with chronic foot ulcers and surgical foot wounds regardless of pathogenesis; 5) NPWT, whether modified or commercial negative pressure devices, compared with standard dressing changes such as various advanced wound dressings and conventional moist gauze; 6) final indicators, in which the primary outcome is the rate of complete ulcer healing and complete wound closure defined as 100\% re-epithelialization without drainage or dressing requirements, and the secondary outcomes included ulcer healing time, change in ulcer size, granulation tissue formation, quality of life, patient satisfaction, resource use, amputation rate, and treatment-related adverse effects (edema, infection, pain, bleeding). Exclusion criteria were as follows: 1) no RCT was performed; 2) NPWT (VAC) was not compared with standard dressing changes; 3) the study did not show corresponding outcomes.

\section{Quality assessment and data collection}

Two reviewers ( $\mathrm{Si}$ Liu, Chao-zhu He) independently assessed the quality of each included study and extracted relevant data; differing opinions were resolved through discussion or a third reviewer's judgment. The reviewers extracted the following information from every included RCT: first author; publication year; study design and size; demographic characteristics of participants; ulcer size, location, and severity; specific implementation of intervention measures (intervention settings, intervention time, the feature of VAC, and details of treatment received by each group); and final indicator measures. We assessed the quality of each included study using the Cochrane Collaboration tool for assessing risk of bias. ${ }^{12}$ This tool addressed six domains including selection bias, performance bias, detection bias, attrition bias, reporting bias, and other bias.

\section{Statistical analysis}

We assessed all data using Revman 5.3 software. First, we conducted the chi-square test to determine whether there was 
heterogeneity among the studies. A result of $P>0.1, I^{2}<50 \%$ indicated no significant heterogeneity between studies; in this case, we used the fixed-effects model for analysis. However, if $P<0.1, I^{2} \geq 50 \%$ and in the absence of clinical heterogeneity, we chose the random-effects model. If $P<0.1$ and we were unable to judge the source of heterogeneity, we used descriptive analysis. We calculated a weighted mean difference (WMD) and 95\% confidence intervals (CIs) for continuous variables and calculated the relative risk (RR) and 95\% CI for dichotomous variables. We considered a twosided $P$ whose value is less than 0.05 to indicate statistical significance. Sensitivity analysis was performed for reduction of DFU area based on the leave-one-out approach.

\section{Results}

\section{Characteristics of studies and assessment}

We retrieved 691 records through database searches. After removing duplicates, we found 587 articles, 549 of which we excluded by reviewing the title and abstract using general criteria, and assessed 37 full-text articles for eligibility. We then excluded 27 studies for the following reasons: did not meet inclusion criteria $(n=4)$; merely a study protocol $(n=1)$; merely a case report $(n=1)$; they were review articles $(n=7)$; they were not an RCT $(\mathrm{n}=10)$; they did not describe diabetic wounds on the foot only $(n=4)$. One article was obtained from a reference list of a retrieved record. We subjected the resulting eleven articles to meta-analysis. ${ }^{13-23}$ Figure 1 shows the specific flow chart. For reasons for final exclusion of 27 studies, see Supplementary material.

Tables 1 and 2 summarize the details of the eleven studies. The eleven RCTs included 1,044 patients. The number of patients in each included article ranged from ten to 342 , the mean ages ranged from 50.2 to 66.5 , and the intervention time ranged from 14 to 112 days. We evaluated the quality of the included RCTs according to the Cochrane reviewers' handbook. ${ }^{12}$ For the included studies, seven of the eleven published articles ${ }^{13-15,17,18,22,23}(63.6 \%)$ described specific randomized methods and processes; we

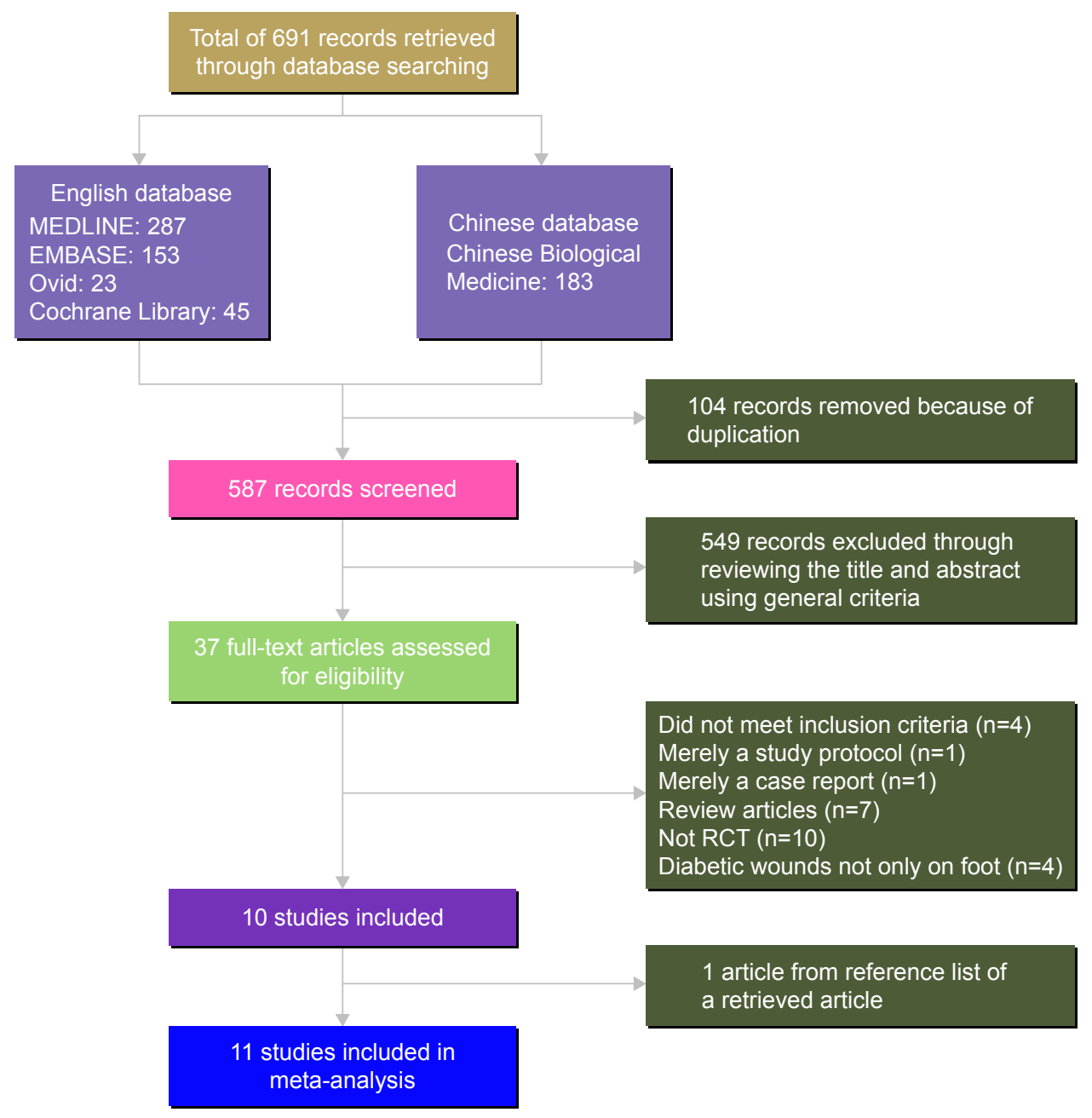

Figure I Flow diagram for identification of studies for inclusion in meta-analysis. Abbreviation: RCT, randomized controlled trial. 


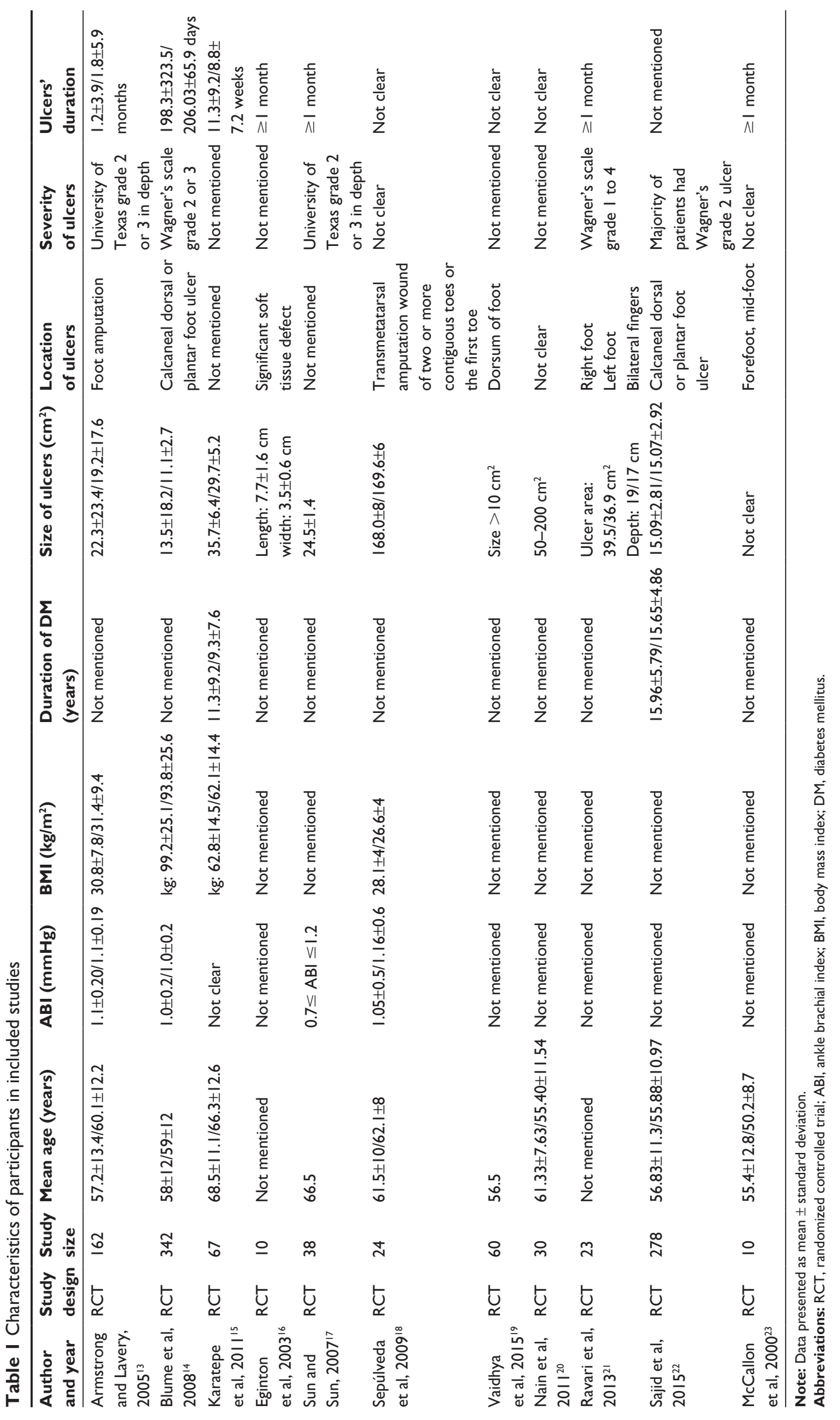




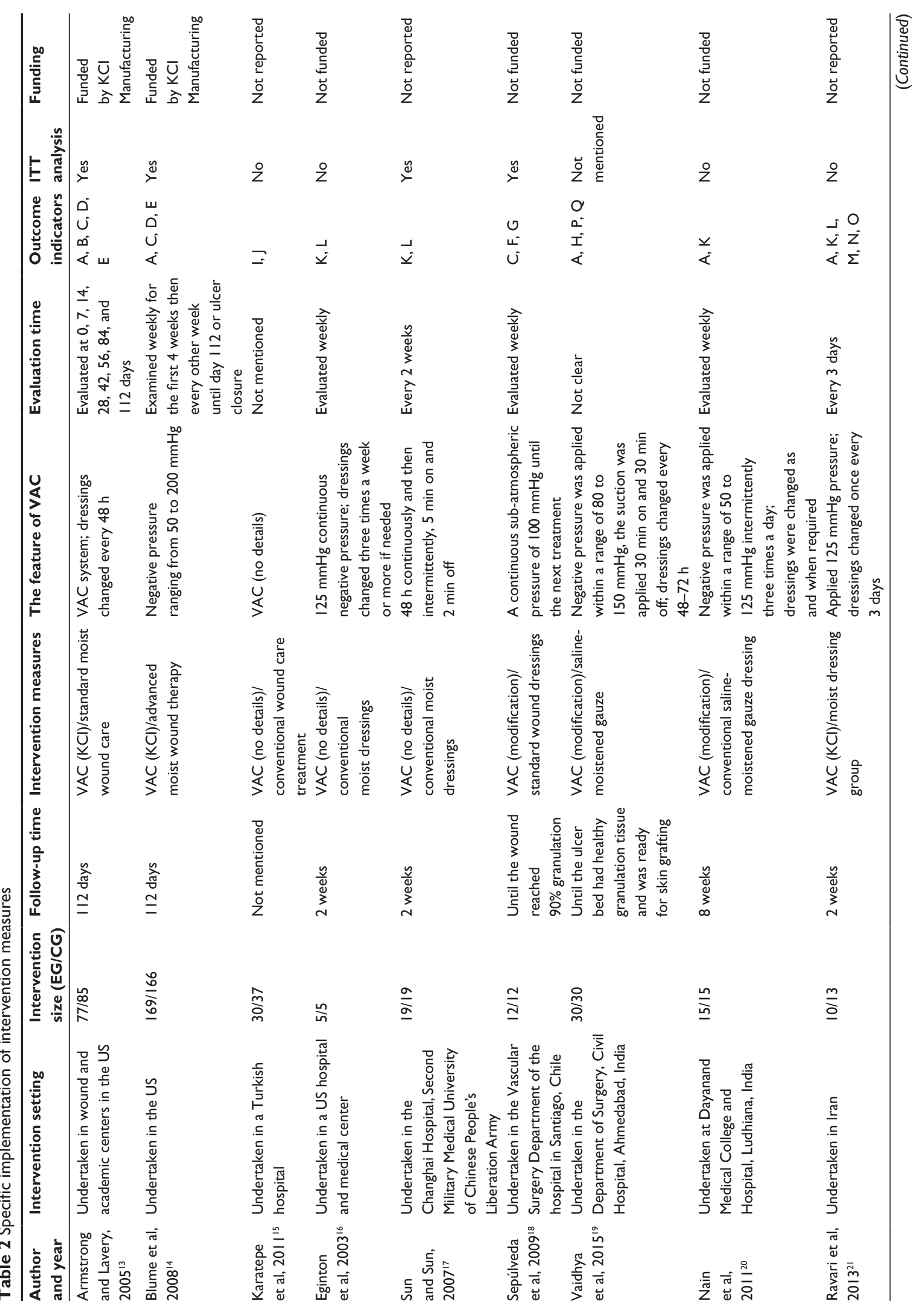




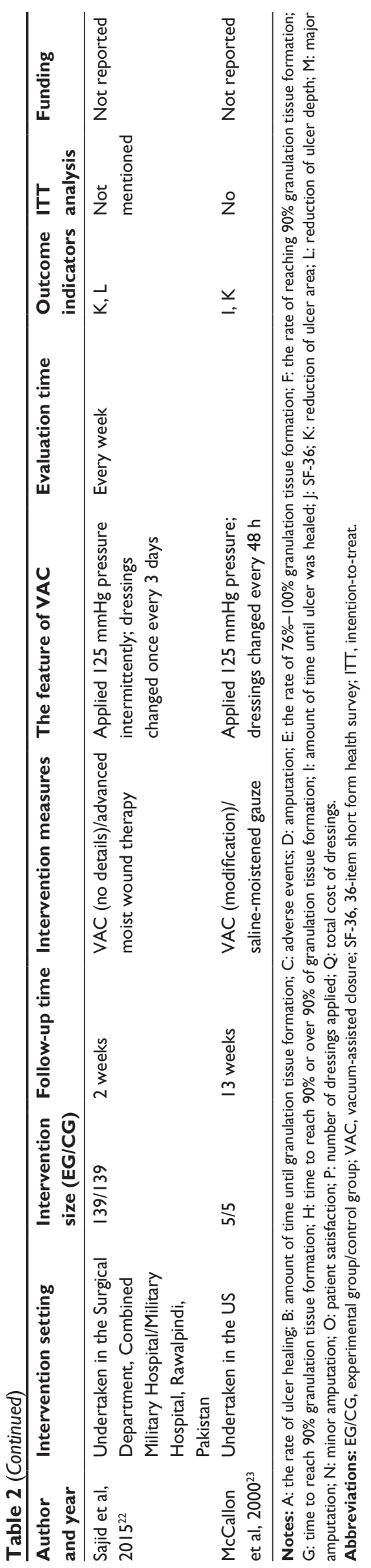

judged one report ${ }^{21}$ to be at high risk of bias for this domain because of randomization based on the date of admission. Three articles ${ }^{13,14,18}(27 \%)$ reported allocation concealment methods. Two articles ${ }^{17,21}$ employed different treatments according to the odevity of case number and date of admission, so we judged them as being at high risk of bias for the allocation concealment domain. It was difficult to achieve a blinded study of participants and personnel in NPWT, but un-blinded health professionals were able to make decisions about closure surgery that could then have resulted in more wound closure or amputation in one group than in the other, ${ }^{24}$ so we classified the risk of bias in this part as unclear. Six articles $^{13,14,16,17,21,22}$ explained the specific tools used for image processing and analysis and had the corresponding data; thus, we may conclude that the outcome assessment was based on the blinded method. Other studies did not contain enough details for us to make a judgment for this domain, so we also judged their risk as unclear. We classified only one study ${ }^{18}$ as having a low risk of bias, because a group independent from the research team, masked the assigned treatment and evaluated the percentage of granulation tissue formation. Five articles ${ }^{13,17-19,23}$ provided information on the loss of cases and the reasons why participants withdrew; another article also provided that information, but the number of cases lost from the experimental and control groups was not clear. Two articles ${ }^{19,20}$ showed some results that had not previously been mentioned, so it was thought to have a risk of publication bias. All studies showed that the baseline data for the experimental group and the control group were comparable. Figures 2 and 3 show the risk of bias in the included studies (details in Supplementary material).

\section{The DFUs' complete healing rate}

Five articles ${ }^{13,14,19-21}$ reported the complete ulcer healing rate. In pooling the data, we found no significant heterogeneity among the five studies $(Q=6.31$, degrees of freedom $[d f]=4, P=0.18 ; I^{2}=37 \%$ ) (Figure 4); therefore, we used a fixed-effects model for the analysis. All reports showed the same results, and the combined RR of 1.48 indicated that the complete ulcer healing rate in the NPWT group was significantly higher than that of the control group (95\% CI: $1.24-1.76, P<0.0001)$.

\section{Time to complete DFU healing}

Four reports ${ }^{13-15,23}$ provided the time to complete DFU healing, but Armstrong et $\mathrm{al}^{13}$ and Blume et $\mathrm{al}^{14}$ offered the estimated time to complete ulcer healing, so we took the other two results into meta-analysis. The two studies showed 


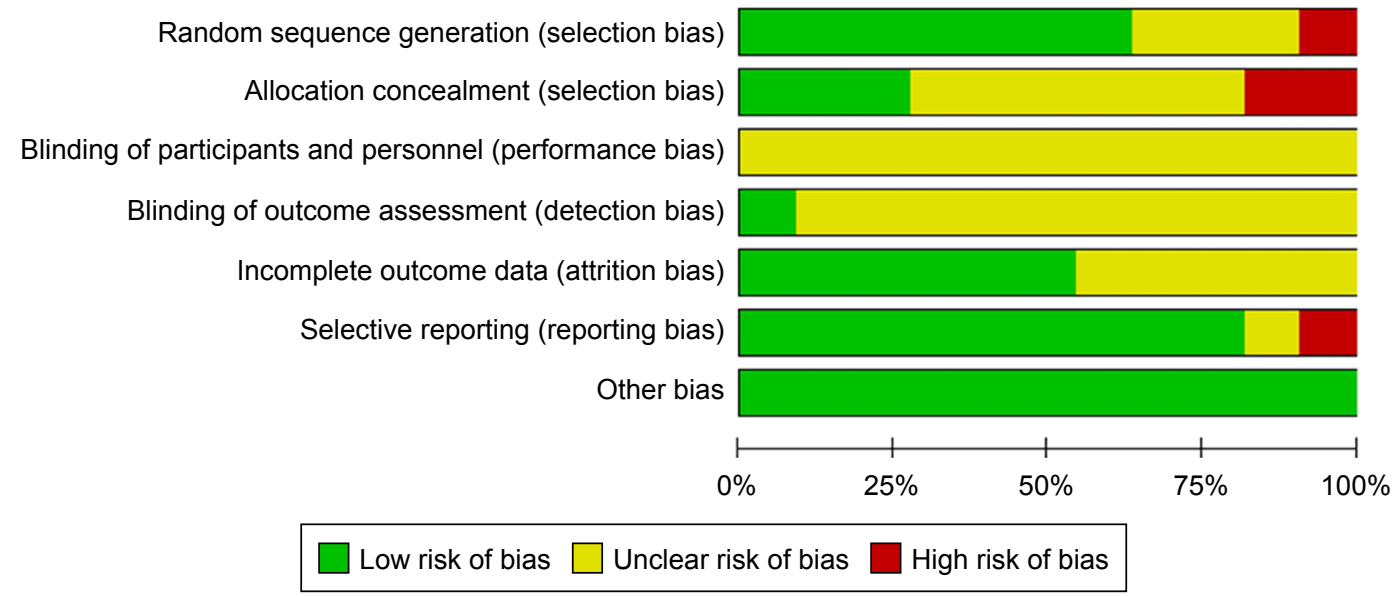

Figure 2 Risk of bias graph.

Note: Review authors' judgments about each risk of bias item presented as percentages across all included studies.

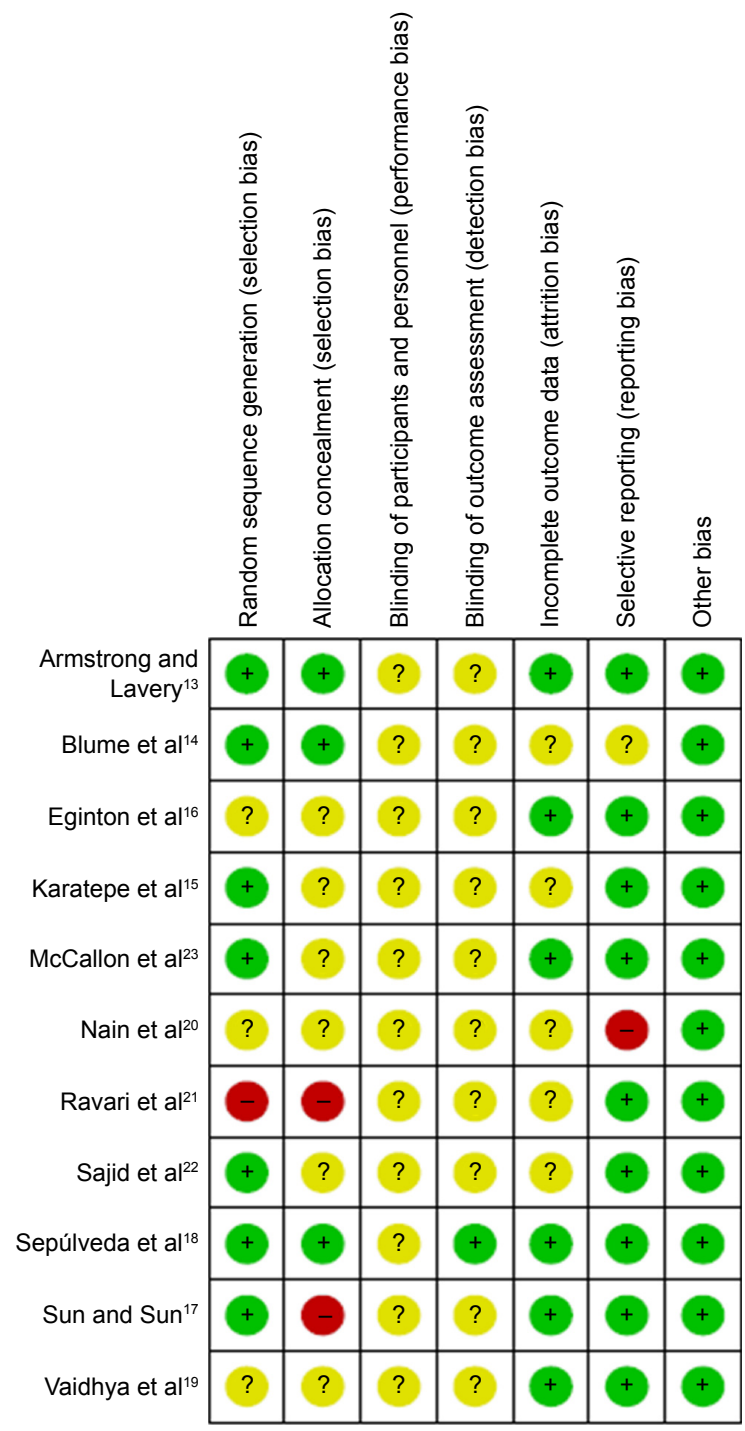

Figure 3 Risk of bias summary.

Note: Review authors' judgments about each risk of bias item for each included study. some homogeneity after we pooled the data $\left(P=0.46 ; I^{2}=0 \%\right)$ (Figure 5). Our meta-analysis result showed that the NPWT group had a shorter time to complete healing of DFUs (mean difference: $-8.07,95 \% \mathrm{CI}:-13.70--2.45, P=0.005)$ compared with that of the standard dressing changes group.

\section{Change in DFUs' size}

Six articles ${ }^{16,17,20-23}$ described a reduction of the DFU area. We found no significant heterogeneity among the six reports after pooling the data $\left(Q=8.30, d f=5, P=0.14 ; I^{2}=40 \%\right.$ ) (Figure 6) and therefore used a fixed-effects model for the analysis. The combined WMD of 12.18 indicated that NPWT more effectively reduced DFUs' area than standard dressing changes (95\% CI: 8.50-15.86, $P<0.00001$ ).

Three articles ${ }^{16,17,21}$ described reduction of DFUs' depth. The three studies showed some homogeneity after we pooled the data $\left(P=0.43 ; I^{2}=0 \%\right.$ ) (Figure 7$)$. The combined WMD of 40.82 indicated that NPWT significantly reduced DFUs' depth in comparison to standard dressing changes ( $95 \%$ CI: $35.97-45.67, P<0.00001)$.

\section{Granulation tissue formation}

Four articles ${ }^{13,14,18,19}$ assessed the granulation tissue formation, but the evaluation results were not unified; therefore, we used descriptive analysis. Armstrong et $\mathrm{al}^{13}$ showed that the time during which $76 \%-100 \%$ of granulation tissue formed in the NPWT group, was shorter than that in the moist dressings change group. Sepúlveda et a $1^{18}$ and Vaidhya et a ${ }^{19}$ provided the average time to reach $90 \%$ or over $90 \%$ of wound granulation tissue formation (18.8 \pm 6 days and $17.2 \pm 3.55$ days, respectively) in the NPWT group; both time periods were shorter than corresponding times in the control group. 


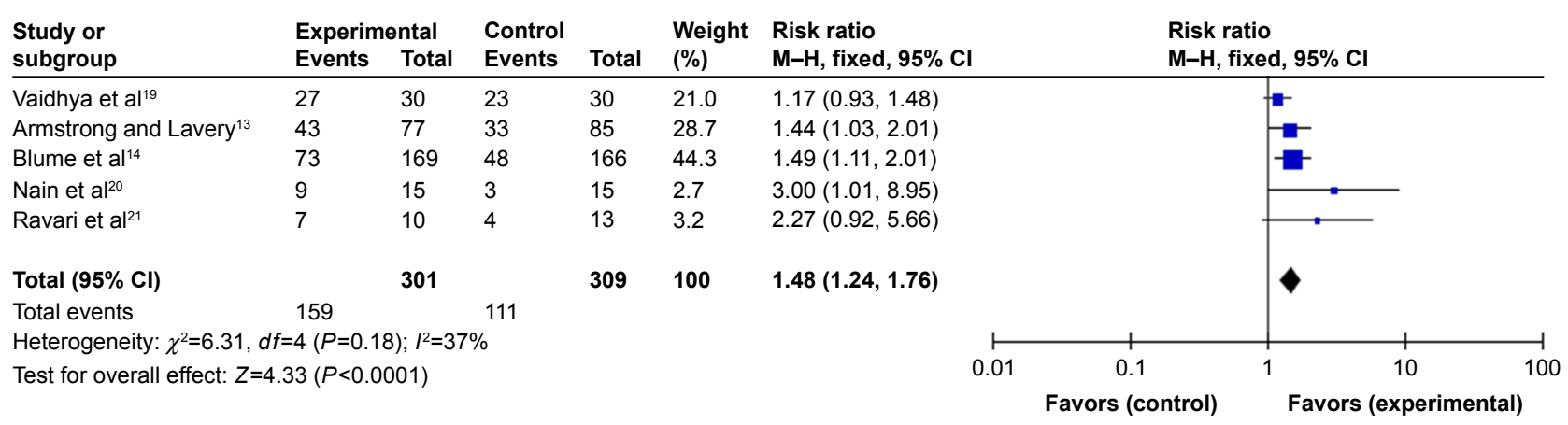

Figure 4 NPWT compared with standard dressing changes, outcome I: the complete DFU healing rate.

Abbreviations: NPWT, negative-pressure wound therapy; DFU, diabetic foot ulcer; $\mathrm{Cl}$, confidence interval; $\mathrm{df}$, degrees of freedom; $\mathrm{M}-\mathrm{H}$, Mantel-Haenszel.

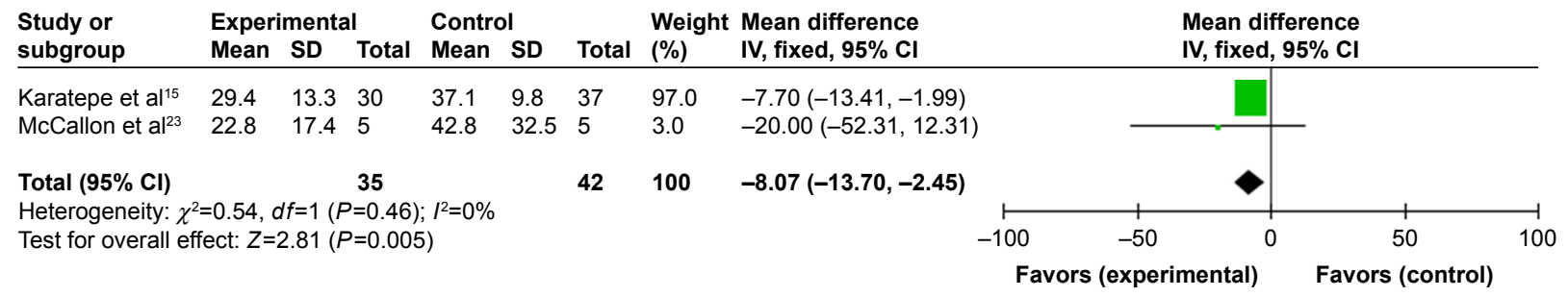

Figure 5 NPWT compared with standard dressing changes, outcome 2: time to complete healing of DFUs.

Abbreviations: NPWT, negative-pressure wound therapy; DFU, diabetic foot ulcer; Cl, confidence interval; $d f$, degrees of freedom; SD, standard deviation; IV, inverse variance.

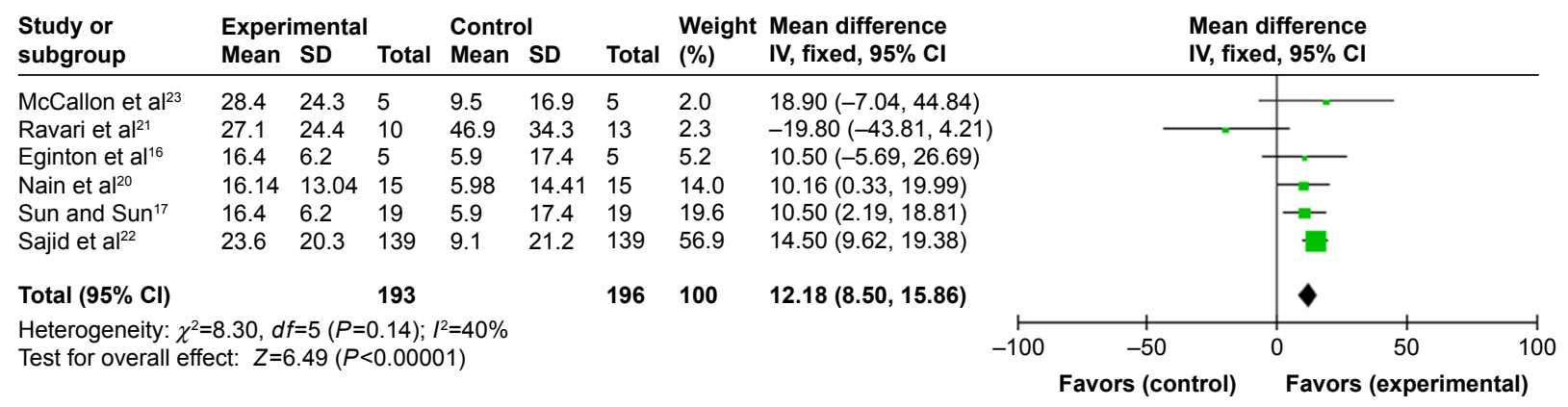

Figure 6 NPWT compared with standard dressing changes, outcome 3: reduction of DFU area.

Abbreviations: NPWT, negative-pressure wound therapy; DFU, diabetic foot ulcer; Cl, confidence interval; df, degrees of freedom; SD, standard deviation; IV, inverse variance.

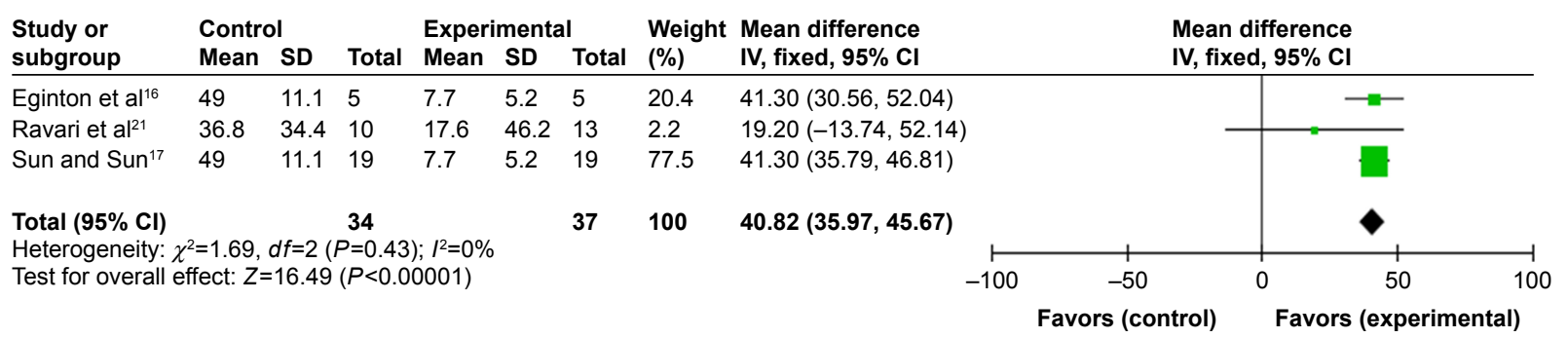

Figure 7 NPWT compared with standard dressing changes, outcome 4: reduction of DFU depth.

Abbreviations: NPWT, negative-pressure wound therapy; DFU, diabetic foot ulcer; Cl, confidence interval; df, degrees of freedom; SD, standard deviation; IV, inverse variance. 


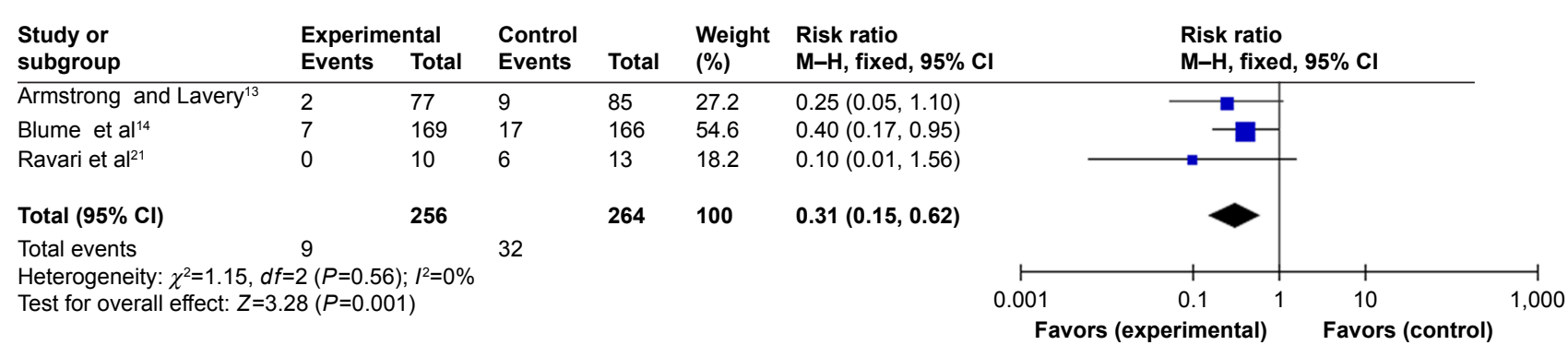

Figure 8 NPWT compared with standard dressing changes, outcome 5: amputation.

Abbreviations: NPWT, negative-pressure wound therapy; Cl, confidence interval; df, degrees of freedom; M-H, Mantel-Haenszel.

\section{Quality of life}

Karatepe et a ${ }^{15}$ had patients fill out the 36 -item short form health survey (SF-36) questionnaire at the beginning of treatment and in the follow-up month, to ascertain whether the patients' quality of life improved after treatment. The SF-36 questionnaire included two sections regarding the patient's physical and mental state. The results showed that the effect of the NPWT treatment was significantly positive for both mental $(P=0.0287)$ and physical $(P=0.004)$ health in comparison to treatment using conventional wound dressing.

\section{Resource use}

Armstrong et $\mathrm{a} \mathrm{l}^{13}$ reported an average total cost per participant of US $\$ 26,972$ in the NPWT group, compared to US\$36,887 in the moist dressing group, with no other information provided. Vaidhya et $\mathrm{al}^{19}$ reported that the mean number of dressings needed to achieve satisfactory healing in the NPWT group was 7.46 \pm 2.25 , compared to $69.8 \pm 11.93(P<0.001)$ for the conventional treatment group. Irrespective of the cost of daily treatment or hospital stay, the average cost of NPWT and of conventional dressing was US\$55 and US\$103 respectively.

\section{Amputation}

Three reports ${ }^{13,14,21}$ provided amputation information. Armstrong et $\mathrm{al}^{13}$ and Blume et $\mathrm{al}^{14}$ analyzed the incidence of re-amputation, Ravari et $\mathrm{al}^{21}$ analyzed the number of patients requiring major and minor amputations. We found no heterogeneity among the three studies after pooling the data $\left(Q=1.15, d f=2, P=0.56 ; I^{2}=0 \%\right.$ ) (Figure 8 ). The combined RR of 0.31 indicated that the incidence of amputation in the NPWT group was lower than in the standard dressing changes group ( $95 \% \mathrm{CI}: 0.15-0.62, P=0.001)$.

\section{Treatment-related adverse events}

Treatment-related adverse DFU events include edema, infection, pain, and bleeding. Infection was the most common adverse event assessed in three RCTs. ${ }^{13,14,16}$ Sepúlveda et $\mathrm{a}^{18}$ included data for bleeding and pain in addition to infection. The result of the meta-analysis indicated that treatment-related adverse events related to DFU showed no significant difference between the NPWT group and the standard dressing changes group (95\% CI: $0.66-1.89$, $P=0.68$ ) (Figure 9).

\section{Sensitivity analysis}

Regarding reduction of the DFU area, when we removed a report that contributed to the final result, the direction and magnitude of the pooled RRs did not vary substantially. This indicated a good reliability of this meta-analysis $(95 \%$ CI: 3.53-14.73, $P=0.001$ ) (Figure 10).

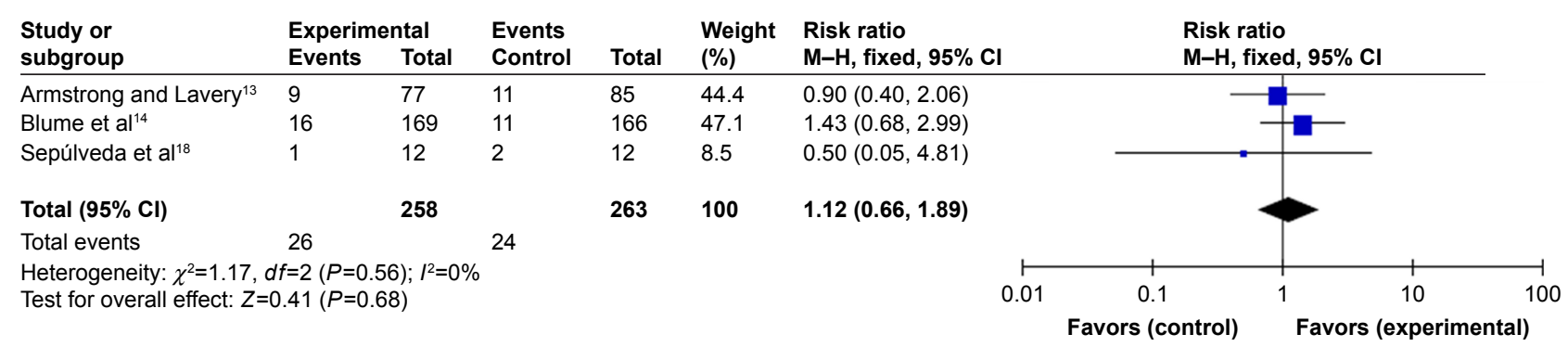

Figure 9 NPWT compared with standard dressing changes, outcome 6: treatment-related adverse events.

Abbreviations: NPWT, negative-pressure wound therapy; $\mathrm{Cl}$, confidence interval; $d f$, degrees of freedom; M-H, Mantel-Haenszel. 


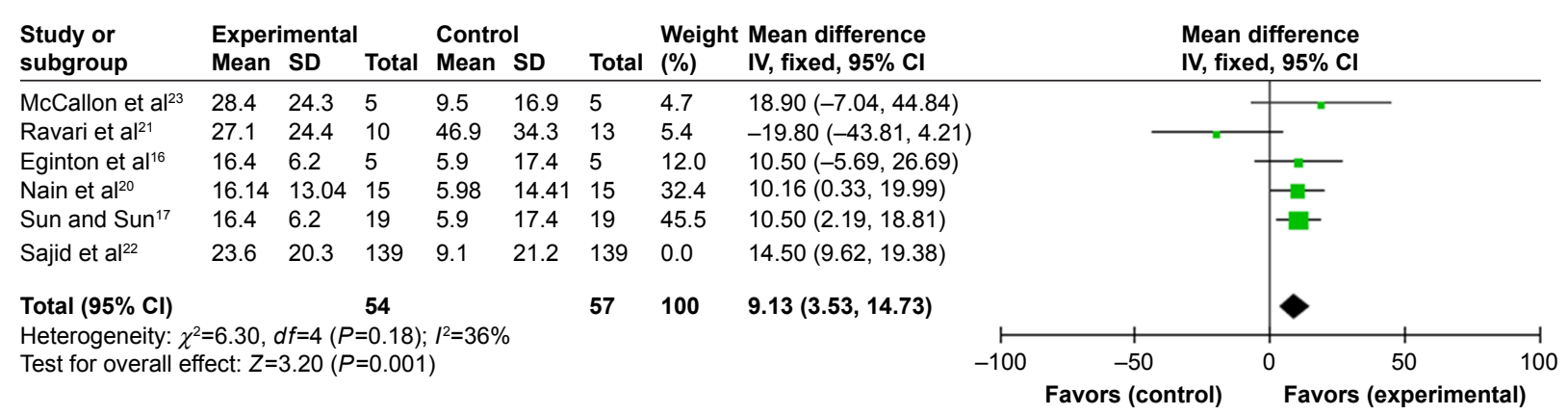

Figure 10 NPWT compared with standard dressing changes, outcome 7: sensitivity analysis.

Abbreviations: NPWT, negative-pressure wound therapy; Cl, confidence interval; df, degrees of freedom; SD, standard deviation; IV, inverse variance.

\section{Discussion}

\section{Evaluation of NPWT efficacy}

In this systematic review and meta-analysis, we found that NPWT facilitated wound granulation formation and complete DFU closure, reduced DFU healing time, and decreased DFU size in comparison with standard dressing changes. Those results were similar to results of prior system reviews. ${ }^{24,25}$ However, another systematic review ${ }^{26}$ concluded that the method of measuring and evaluating ulcer size reduction and complete wound closure may affect the reliability of the results. Therefore, for outcome measures, it is important to focus on the use of blinded measures. Wound bed preparation and granulation tissue formation are also important prerequisites for wound healing. The Patient Outcome Group suggested that the appropriate primary endpoint may not be DFU healing but, rather, percentage granulation tissue formation. ${ }^{27}$ Four articles ${ }^{13,14,18,19}$ assessed granulation tissue formation, and two of them used $90 \%$ or more than $90 \%$ of granulation tissue formation, preparation of re-epithelialization, and skin grafting as endpoints. The evaluation results showed that NPWT could accelerate granulation formation in comparison to standard dressing changes. It is known that foot wounds secondary to amputation are deeper, with exposed bone and tendons and pre-existing infection, and would lead to delayed wound healing. Armstrong et a ${ }^{13}$ enrolled 162 diabetic patients with post-operative wounds to receive NPWT treatment or moist dressing treatment. The rate of complete wound healing for patients receiving NPWT (56\%) was higher than for the moist dressings group (39\%); the median time to reach $76 \%-100 \%$ granulation tissue for patients receiving NPWT (42 days) was less than for the control group (84 days), which suggested that NPWT had the potential to promote more complex and severe wound healing and prepare an adequately granulated wound bed.

\section{Evaluation of NWPT safety}

Treatment-related adverse DFU events include edema, infection, pain, and bleeding. The meta-analysis results showed that NPWT neither increased nor decreased the incidence of treatment-related side effects as compared with the standard dressing change group; which suggested that adverse events related to NPWT were not serious. However, in 2011 the US Food and Drug Administration updated a report on serious complications associated with NPWT and cited 12 deaths and 174 injuries since 2007. ${ }^{11}$ Ren and Li reported sepsis in a burns patient treated with NPWT. ${ }^{28}$ It should be noted that acute hemorrhages caused all of the deaths because large, exposed blood vessels and bleeding were ignored. Meanwhile, some of these serious adverse events occurred at home or in a long-term care facility, where the patients, nurses, and home care providers might not have received adequate training to do NPWT properly. In the eleven RCTs that we included in our meta-analysis, the intervention settings were hospitals or wound centers where professionals are familiar with NPWT indications and adhere to treatment guidelines. ${ }^{29}$ This may be why serious complications did not occur in the studies we reviewed in our meta-analysis. DFUs are a leading cause of non-traumatic foot amputation; Armstrong et $\mathrm{al}^{13}$ reported that the number of patients who received NPWT treatment were a quarter less likely to need re-amputation compared to controls. The result of our meta-analysis also indicated that NPWT could effectively reduce the occurrence of amputation. The rate of amputation decreased in the NPWT treatment group and is attributed to faster removal of infectious material, better preparation of granulated wound bed, and more rapid healing.

\section{Evaluation of NPWT cost-effectiveness}

A post hoc retrospective analysis indicated that for patients with DFUs who achieved complete wound closure, the median cost per $1 \mathrm{~cm}^{2}$ of closure was US\$1,227 with NPWT and US\$1,695 with advanced moist wound therapy, which showed greater cost-effectiveness in the NPWT group for treating recalcitrant wounds. ${ }^{30}$ Two analyses ${ }^{31,32}$ based on economic models also concluded that, compared to patients 
treated with advanced wound care, patients treated with VAC therapy had increased quality-adjusted life years and a higher healing rate at a lower cost. Vaidhya et al ${ }^{19}$ concluded that the mean dressings and total cost of dressings needed to achieve satisfactory healing in the NPWT group, were less than for the conventional dressing changes group. However, in this RCT, the VAC was modified to the standard KCI VAC therapy kit, so subsequent RCTs are needed to evaluate the cost of commercial VAC NPWT for treating DFUs. Moreover, considering the actual situation of medical resources available in developing countries, a modified NPWT device may be a future research direction for NPWT experiments in resource-poor settings.

\section{Evaluation of other aspects of NPWT}

One $\mathrm{RCT}^{17}$ evaluated quality of life using the SF-36 questionnaire, which suggested that NPWT remarkably improved the quality of life of DFU patients. Another RCT, in which no amputation was performed, ${ }^{21}$ evaluated patient satisfaction by that measure, indicating that patients in the NPWT group were more satisfied. However, we would prefer to survey patients rather than relying on a secondary outcome to assess patient satisfaction.

\section{Limitations}

From the details of included studies, important information was not fully available. Only four articles ${ }^{13,14,17,18}$ offered data related to the ankle brachial index (ABI), even though $\mathrm{ABI}$ measurement is a simple and effective method of judging lower limb vascular disease to determine whether amputation is necessary. ${ }^{33}$ Two studies ${ }^{15,22}$ provided the duration of DM, which could influence the peripheral neuropathy leading to the formation of DFUs. ${ }^{34}$ It was reported that body mass was significantly associated with pressure in the mid-foot models. ${ }^{35}$ Two articles calculated average weight, and another two calculated body mass index, whereas no relevant details about local pressure on the foot were provided in the remaining seven studies. Stratified randomization was not performed for the severity of DFUs, thus, the patient characteristics in each group were not balanced. Meanwhile, there were many other influencing factors, including the relatively small sample sizes, insufficient description of methodologic details, inadequate follow-up time, and so on, which can result in clinical heterogeneity. Finally, because we retrieved only published literature, the document collection may be incomplete.

\section{Conclusion}

This meta-analysis of eleven RCTs extends support for the use of NPWT in the treatment of DFUs and post-operative wounds in diabetic patients. Additional robust RCT research is necessary to solidify support for the treatment.

\section{Acknowledgment}

The authors would like to thank Ming Huang for guidance in terms of writing and submission of the paper.

\section{Disclosure}

The authors report no conflicts of interest in this work.

\section{References}

1. Heublein H, Bader A, Giri S. Preclinical and clinical evidence for stem cell therapies as treatment for diabetic wounds. Drug Discov Today. 2015;20(6):703-717.

2. Toosizadeh N, Mohler J, Armstrong DG, Talal TK, Najafi B. The influence of diabetic peripheral neuropathy on local postural muscle and central sensory feedback balance control. PLoS One. 2015; 10(8): 0135255.

3. Boulton AJ. The diabetic foot. Medicine. 2010;38(12):644-648.

4. Setacci F, Sirignano P, De Donato G, et al. Primary amputation: is there still a place for it. J Cardiovasc Surg (Torino). 2012;53(1):53-59.

5. Kvitkina T, Narres M, Claessen H, et al. Incidence of lower extremity amputation in the diabetic compared to the non-diabetic population: a systematic review protocol. Syst Rev. 2015;4:74.

6. Rice JB, Desai U, Cummings AK, Birnbaum HG, Skornicki M, Parsons NB. Burden of diabetic foot ulcers for medicare and private insurers. Diabetes Care. 2014;37(3):651-658.

7. Cavanagh P, Attinger C, Abbas Z, Bal A, Rojas N, Xu ZR. Cost of treating diabetic foot ulcers in five different countries. Diabetes Metab Res Rev. 2012;28 (Suppl 1):S107-S111.

8. Söylemez MS, Özkan K, Kılıç B, Erinc S. Intermittent negative pressure wound therapy with instillation for the treatment of persistent periprosthetic hip infections: a report of two cases. Ther Clin Risk Manag. 2016;12:161-166.

9. Vassallo IM, Formosa C. Comparing calcium alginate dressings to vacuum-assisted closure: a clinical trial. Wounds. 2015;27(7): 180-190.

10. Mody GN, Nirmal IA, Duraisamy S, Perakath B. A blinded, prospective, randomized controlled trial of topical negative pressure wound closure in India. Ostomy Wound Manage. 2008;54(12):36-46.

11. Safety Communications-UPDATE on serious complications associated with negative pressure wound therapy systems: FDA safety communication; 2011. Available from: https://www.fda.gov/MedicalDevices/ Safety/AlertsandNotices/ucm244211.htm. Accessed April 7, 2017.

12. Higgins JP, Green S, editors. Cochrane Handbook for Systematic Reviews of Interventions 5.1.0. The Cochrane Collaboration; 2011.

13. Armstrong DG, Lavery LA; Diabetic Foot Study Consortium. Negative pressure wound therapy after partial diabetic foot amputation: a multicentre, randomised controlled trial. Lancet. 2005;366(9498): 1704-1710.

14. Blume PA, Walters J, Payne W, Ayala J, Lantis J. Comparison of negative pressure wound therapy using vacuum-assisted closure with advanced moist wound therapy in the treatment of diabetic foot ulcers: a multicenter randomized controlled trial. Diabetes Care. 2008; 31(4):631-636.

15. Karatepe O, Eken I, Acet E, et al. Vacuum assisted closure improves the quality of life in patients with diabetic foot. Acta Chir Belg. 2011;111(5):298-302.

16. Eginton MT, Brown KR, Seabrook GR, Towne JB, Cambria RA. A prospective randomized evaluation of negative-pressure wound dressings for diabetic foot wounds. Ann Vasc Surg. 2003;17(6):645-649.

17. Sun JW, Sun JH. Vacuum assisted closure technique for repairing diabetic foot ulcers: analysis of variance by using a randomized and doublestage crossover design. J Clin Rehab Tissue Eng Res. 2007;11(44): 8908-8911. 
18. Sepúlveda G, Espíndola M, Maureira M, et al. Curación asistida por presión negativa comparada con curación convencional en el tratamiento del pie diabético amputado. Ensayo clínico aleatorio. [Negative-pressure wound therapy versus standard wound dressing in the treatment of diabetic foot amputation. A randomised controlled trial]. Cir Esp. 2009;86(3):171-177. Spanish.

19. Vaidhya N, Panchal A, Anchalia MM. A new cost-effective method of NPWT in diabetic foot wound. Indian J Surg. 2015;77 (Suppl 2): 525-529.

20. Nain PS, Uppal SK, Garg R, Bajaj K, Garq S. Role of negative pressure wound therapy in healing of diabetic foot ulcers. J Surg Tech Case Rep. 2011;3(1):17-22.

21. Ravari H, Modaghegh MH, Kazemzadeh GH, et al. Comparison of vacuum-assisted closure and moist wound dressing in the treatment of diabetic foot ulcers. J Cutan Aesthet Surg. 2013;6(1):17-20.

22. Sajid MT, Mustafa Qu, Shaheen N, Hussain SM, Shukr I, Ahmed M. Comparison of negative pressure wound therapy using vacuumassisted closure with advanced moist wound therapy in the treatment of diabetic foot ulcers. J Coll Physicians Surg Pak. 2015;25(11): 789-793.

23. McCallon SK, Knight CA, Valiulus JP, Cunningham MW, McCulloch JM, Farinas LP. Vacuum-assisted closure versus salinemoistened gauze in the healing of postoperative diabetic foot wounds. Ostomy Wound Manage. 2000;46(8):28-32, 34.

24. Dumville JC, Hinchliffe RJ, Cullum N, et al. Negative pressure wound therapy for treating foot wounds in people with diabetes mellitus. Cochrane Database Syst Rev. 2013;10(10):CD010318.

25. Zhang J, Hu ZC, Chen D, Guo D, Zhu JY, Tang B. Effectiveness and safety of negative-pressure wound therapy for diabetic foot ulcers: a meta-analysis. Plast Reconstr Surg. 2014;134(1):141-151.

26. Peinemann F, Sauerland S. Negative-pressure wound therapy: systematic review of randomized controlled trials. Dtsch Arztebl Int. 2011; 108(22):381-389.
27. Gottrup F, Apelqvist J, Price P; European Wound Management Association Patient Outcome Group. Outcomes in controlled and comparative studies on non-healing wounds: recommendations to improve the quality of evidence in wound management. J Wound Care. 2010; 19(6):237-268.

28. Ren H, Li Y. Severe complications after negative pressure wound therapy in burned wounds: two case reports. Ther Clin Risk Manag. 2014;10:513-516

29. Schintler MV. Negative pressure therapy: theory and practice. Diabetes Metab Res Rev. 2012;28 Suppl 1:72-77.

30. Driver VR, Blume PA. Evaluation of wound care and health-care use costs in patients with diabetic foot ulcers treated with negative pressure wound therapy versus advanced moist wound therapy. J Am Podiatr Med Assoc. 2014;104(2):147-153.

31. Flack S, Apelqvist J, Keith M, Trueman P, Williams D. An economic evaluation of VAC therapy compared with wound dressings in the treatment of diabetic foot ulcers. $J$ Wound Care. 2008;17(2):71-78.

32. Whitehead SJ, Forestbendien VL, Richard JL, Halimi S, Van GH, Trueman P. Economic evaluation of Vacuum Assisted Closure ${ }^{\circledR}$ therapy for the treatment of diabetic foot ulcers in France. Int Wound J. 2011;8(1):22-32.

33. Al-Rubeaan K, Al Derwish M, Ouizi S, et al. Diabetic foot complications and their risk factors from a large retrospective cohort study. PLoS One. 2015;10(5): $\mathrm{e} 0124446$

34. Alavi A, Sibbald RG, Mayer D, et al. Diabetic foot ulcers: Part I. Pathophysiology and prevention. J Am Acad Dermatol. 2014;70(1): 1.e1-e18.

35. Barn R, Waaijman R, Nollet F, Woodburn J, Bus SA. Predictors of barefoot plantar pressure during walking in patients with diabetes, peripheral neuropathy and a history of ulceration. PLoS One. 2015; 10(2):e0117443.
Therapeutics and Clinical Risk Management

\section{Publish your work in this journal}

Therapeutics and Clinical Risk Management is an international, peerreviewed journal of clinical therapeutics and risk management, focusing on concise rapid reporting of clinical studies in all therapeutic areas, outcomes, safety, and programs for the effective, safe, and sustained use of medicines. This journal is indexed on PubMed Central, CAS,

\section{Dovepress}

EMBase, Scopus and the Elsevier Bibliographic databases. The manuscript management system is completely online and includes a very quick and fair peer-review system, which is all easy to use. Visit $\mathrm{http}: / / \mathrm{www}$.dovepress.com/testimonials.php to read real quotes from published authors. 